\title{
Tunable Integrated Optical Filters Based on Sapphire Microspheres and Liquid Crystals
}

\author{
Giovanni Gilardi ${ }^{\mathrm{a}, \mathrm{c}}$, Hasan Yılmaz ${ }^{\mathrm{b}}$, Mohammed Sharif Murib ${ }^{\mathrm{b}}$, Rita Asquini ${ }^{\mathrm{c}}$, \\ Antonio d'Alessandro, ${ }^{\mathrm{a}, \mathrm{c}}$, Ali Serpengüzel ${ }^{\mathrm{b}}$ and Romeo Beccherelli ${ }^{{ }^{*} \mathrm{a}}$ \\ ${ }^{a}$ Consiglio Nazionale delle Ricerche - Istituto per la Microelettronica e Microsistemi (CNR-IMM) \\ Via del Fosso del Cavaliere, 100 - 00133 Rome - Italy \\ ${ }^{\mathrm{b}}$ Koç University, Microphotonics Research Laboratory, Department of Physics \\ Rumelifeneri Yolu, Sarıyer, Istanbul 34450 Turkey \\ ${ }^{\mathrm{c}}$ Department of Electronic Engineering, University of Rome "La Sapienza" \\ Via Eudossiana, 1800184 Rome - Italy
}

\begin{abstract}
We present an integrated optical narrowband electrically tunable filter based on the whispering gallery modes of sapphire microspheres and double ion-exchanged channel BK7 glass waveguides. Tuning is provided by a liquid crystal infiltrated between the spheres and the glass substrate. By suitably choosing the radii of the spheres and of the circular apertures, upon which the spheres are positioned, arrays of different filters can be realized on the same substrate with a low cost industrial process. We evaluate the performance in terms of quality factor, mode spacing, and tuning range by comparing the numerical results obtained by the numerical finite element modeling approach and with the analytical approach of the Generalized Lorenz-Mie Theory for various design parameters. By reorienting the LC in an external electrical field, we demonstrate the tuning of the spectral response of the sapphire microsphere based filter. We find that the value of the mode spacing remains nearly unchanged for the different values of the applied electric field. An increase of the applied electric field strength, changes the refractive index of the liquid crystal, so that for a fixed geometry the mode spacing remains unchanged.
\end{abstract}

Keywords: Coupler, Filter, Modulation, Microcavity, Mie Scattering, Spectroscopy, Liquid crystals

\section{INTRODUCTION}

Dielectric microspheres have optical modes of propagation, i.e., whispering gallery modes (WGM's), which correspond to the resonance condition for which the light beam travels, through total internal reflection (TIR), in proximity of the surface of the dielectric sphere. In 1912, Lord Rayleigh published "The problem of the whispering gallery", in which he demonstrated the very efficient propagation of acoustic waves along the internal surface of the dome of St. Paul's Cathedral in London. For a large integer number $\mathrm{N}$ of reflections on the internal surface of the wall, the inner volume is not relevant to the propagation, as the wave travels near the surface. The same principle of TIR applies to optical WGM resonators. In general, the larger the N, the larger the quality factor Q. For a very large and perfectly smooth sphere, Q would ideally be limited by the absorption loss in the material. If the sphere is made of a material with low absorption losses, the high Q and the elevated spatial confinement of the wave render such device of great interest for the possible applications in various fields like photonics and sensors.

Initially, polymer ${ }^{2,3}$ and glass ${ }^{4}$ spheres placed on glass optical fiber half couplers have been excited with evanescent wave coupling in the visible. Later, tapered optical fibers ${ }^{5}$ and channel waveguides ${ }^{6}$ have also been used for coupling light into the spheres. Applications of microsphere encompass microlasers ${ }^{7}$, filters based glass ${ }^{8}$ and silicon ${ }^{9}$ microspheres, switches ${ }^{10}$, high resolution spectroscopy applications ${ }^{11}$ and Raman sources ${ }^{12}$. Silicon microspheres have been used for optical modulation in the near-infrared ${ }^{13}$.

Liquid crystals (LC) can provide means for wide tuning of integrated optical filters ${ }^{14,15,16}$. Very recently, we have proposed a narrowband electrically tunable filter based on a sapphire microsphere, a glass optical waveguide and LC as

* romeo.beccherelli@artov.imm.cnr.it; Phone +390649934538 Fax +390649934066

Silicon Photonics and Photonic Integrated Circuits II, edited by Giancarlo Cesare Righini,

Proc. of SPIE Vol. 7719, 77191E · (C) 2010 SPIE · CCC code: 0277-786X/10/\$18 · doi: 10.1117/12.855978 
tuning medium for telecommunication applications in the near-infrared ${ }^{17}$. The LC is infiltrated in the volume between an optical waveguide and the microsphere. By reorienting the LC in an applied electrical field, we have demonstrated the electrical tuning of the spectral response of a WGM filter. Our work was soon followed by a LC tuned microdisk ${ }^{18 .}$

In this work, we briefly recall the device structure and compare the numerical results obtained by the numerical finite element modeling approach with the analytical approach of the Generalized Lorenz-Mie Theory (GLMT) ${ }^{19}$ for various design parameters.

\section{DEVICE FABRICATION PROCESS}

The fabrication process is conceived with the objective of an easy realization and the minimal sensitivity regarding the variations of the geometric parameters. We first start by realizing a double ion-exchange single mode waveguide in borosilicate krone 7 (BK7) glass. The procedure is carried out in a simple furnace based on a fluidized alumina bath which provides temperature control accuracy of $\pm 1{ }^{\circ} \mathrm{C}$. In a first ion-exchange process, a pre-cleaned bare BK7 glass wafer is immersed into a pure $\mathrm{KNO}_{3}$ melt at $400{ }^{\circ} \mathrm{C}$ for 80 minutes. This $\mathrm{K}^{+} \leftrightarrow \mathrm{Na}^{+}$ion exchange uniformly raises the refractive index close to the surface. Hence, a $200-\mathrm{nm}$-thick Al film is deposited on one side of the wafer and an aperture is photolithographically defined to produce a spatially selective protective mask. The masked wafer is then dipped into a molten 1:10 mol:mol mixture of $\mathrm{AgNO}_{3}: \mathrm{NaNO}_{3}$ at $330{ }^{\circ} \mathrm{C}$ for 5 hours. This localized $\mathrm{Ag}^{+} \leftrightarrow \mathrm{Na}^{+}$ion exchange selectively raises the refractive index in the unmasked glass, thus resulting in a waveguide. The same Al film is photolithographically patterned a second time to define controlling electrodes located sufficiently far away from the waveguides to prevent optical absorption losses. These are further reduced to $\approx 1 \mathrm{~dB} / \mathrm{cm}$ by a 24 hour annealing at 300 ${ }^{\circ} \mathrm{C}$. Finally end-facets are polished to allow fiber butt-coupling.

The processed BK7 glass wafer with the ion exchange waveguide, is then fully covered with polydimethylsiloxane (PDMS), with a refractive index of $1.406 @ 1550 \mathrm{~nm}$. For a diffused waveguide from a $6 \mu \mathrm{m}$ wide aperture in the Al film and a $4 \mu \mathrm{m}$ thick PDMS cover layer, the effective refractive index of the single TE mode is calculated as 1.5108. The refractive index profile is shown in Figure 1.

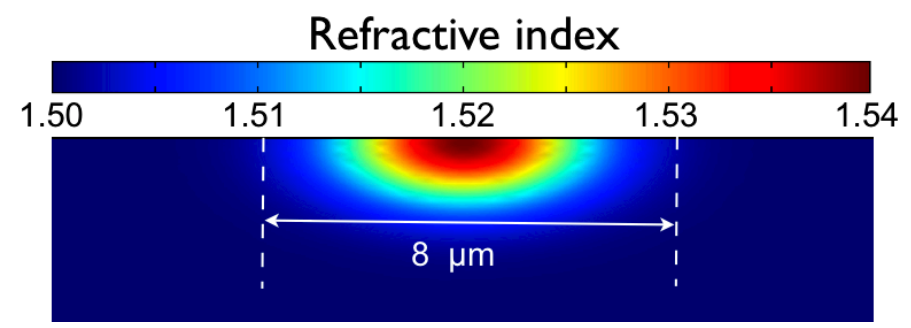

Figure 1. Refractive index profile of the double ion exchange glass waveguide.

The PDMS layer is then selectively patterned to host the sphere, to allow the LC to fill the volume comprised in between the sphere and the glass and to expose the electric contacts. The combination of the PDMS thickness and the diameter of the cylindrical aperture accurately determine the distance between the waveguide and the sphere. Using identical microspheres, various diameters for the cylindrical apertures allow the production of different filters on a single glass substrate. After the realization of the apertures, a thin layer of Nylon 6 is deposited on the entire substrate and rubbed with a velvet cloth along the propagation direction $z$ in order to promote uniform alignment of the LC. Due to the thin (only $4 \mu \mathrm{m}$ ) layer of PDMS and the typical length of the velvet cloth fiber used for rubbing (100s of $\mu \mathrm{m}$ ), the rubbing process works well also at the bottom of the cylindrical aperture. The presence of Nylon 6 above the PDMS layer is fully irrelevant as there is no optical field in it. The sapphire microspheres are positioned (see Figure 2) over the reservoir and glued in place. A LC is infiltrated in its isotropic phase by capillary action through the lateral channels, that reach the cylindrical apertures and let slowly cool to room temperature to obtain a well ordered nematic phase. Finally, the channels are sealed with glue in order to avoid the spillage of the LC. The glue is applied in two stages. In a first stage tiny droplets are placed at four points $( \pm \pi / 4, \pm 3 \pi / 4$ with respect to $z$ axis) to hold the sphere in place. This prevents coupling to the propagating direction and leaves the filling channels free. After the cylindrical reservoir is filled, further glue is used to seal the filling channels. The lateral positioning of the channels prevents the glue from mixing with the LC along the optical path, thus avoiding scattering centers along the propagation direction ${ }^{20}$. The glue may partially mix 
with the LC and forms droplets within a volume having length-scale comparable to the reservoir (few unit $\mu \mathrm{m}$ ). However, these droplets are significantly far away from the LC materials relevant for propagations.

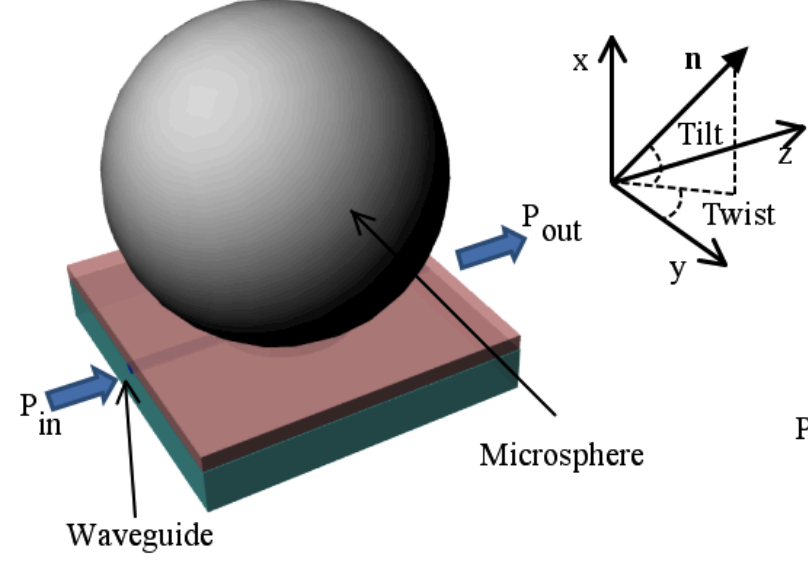

(a)

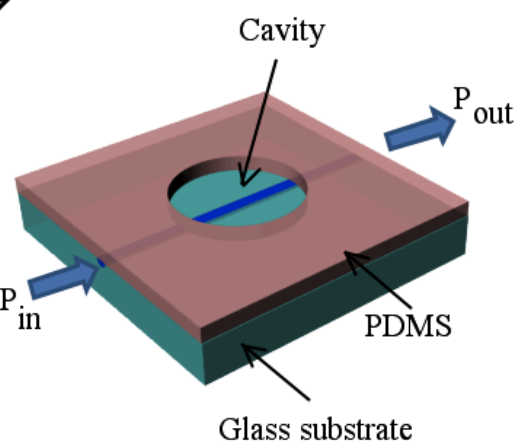

Glass substrate

(b)

Figure 2. Schematic view of the device with (a) and without (b) the sphere in place, showing the cavity to be infiltrated by the LC.

\section{MODELING}

We model here our device with a twofold approach: (i) numerically by a Finite Element Method (FET) and (ii) analytically by Generalized Lorenz-Mie Theory (GLMT). We consider a microsphere described by equation (1).

$$
\left(x-x_{0}\right)^{2}+y^{2}+z^{2}=s^{2}
$$

Its center is raised above the waveguiding medium by $x_{o}=s+d$, where $s$ is the radius ( $37.5 \mu \mathrm{m}$ in this case) and $d$ is the sphere to substrate distance. The radius of the cylindrical reservoir is given in equation (2).

$$
r=\sqrt{2[d(h-s)+h s]-h^{2}-d^{2}}
$$

In equation (2) $h$ is the height the PDMS layer ( $8 \mu \mathrm{m}$ in the present case). When the sphere is in direct contact with the substrate $(d=0)$, the reservoir radius is $23 \mu \mathrm{m}$ which is conveniently obtained by optical lithography. For these design parameters, the LC extends over $10.56 \%$ of the circumference and $10.67 \%$ of the surface of the sphere.

The transmission of the optical power from the input port to the output port is controlled with an applied voltage (V), which determines the tilt and twist angles of the LC molecular director $n$. Tilt and twist angles are defined in Figure 2. The position of the electrodes at the sides of the waveguide and the rubbing of the alignment layer along the propagation axis $z$ makes sure that the electric field mainly acts on the twist angle, forcing the LC molecular director $y$ to rotate only in the $y z$ plane. It is experimentally found that in waveguides fabricated with the process described here only one transverse electric (TE) mode propagates. This TE mode of the waveguide will couple to the TE WGMs of the microsphere. The transverse magnetic (TM) WGMs will not be excited. Hence, electric field control affects mainly the TE polarized light, i.e., the lightwave having its electric field vector along the $y$ direction. Refractive indices of glass substrate and waveguide, PDMS, sapphire and air are not subject to change under an applied voltage. This index is a function of "twist", one of the two characteristic angles universally used to describe orientation of LC. Twist is defined only in the volume filled by the LC. Twist varies with $x, y, z$ (i.e. $\operatorname{twist}=\operatorname{twist}(x, y, z))$ under the applied voltage. The effective refractive index of the waveguide depends on the combination of $n_{T E}$ (twist) and the refractive indices of the other materials. Hence, the waveguide is non-uniform, with and effective refractive index varying along $z$. An increase in $n_{T E}$ (twist) results in longer penetration depth and larger optical field in the LC. The larger the penetration depth, the larger the power coupled to the microsphere. The increase of $n_{T E}$ (twist) modifies the relative refractive index of the microsphere, therefore modifying the spectral position of the WGMs. 
In the standard near-infrared telecommunication band of $1550 \mathrm{~nm}$, the effective refractive index of the fundamental mode of the waveguide is 1.5108 , the sapphire sphere has a refractive index of 1.7, the liquid crystal chosen, the well known cyanobiphenyl mixture E7, has an ordinary and extraordinary refractive index of 1.5 and 1.69 , respectively. As the GLMT does not handle easily anisotropic media, we here simplify the anisotropic problem and model the liquid crystal as an isotropic medium for both approaches with $n=n_{T E}$ (twist). This simplification does not significantly alter the accuracy of our results. We excite the waveguide with the TE polarizations which sees only the ordinary refractive index of the LC and we consider only minor twist perturbations from the perfect planar alignment, i.e. minor increase in the isotropic refractive index. Fully anisotropic calculations were previously reported ${ }^{17}$.

\subsection{Numerical modeling}

The device has been numerically simulated with a Finite Element Method model specifically implemented by us in COMSOL Multiphysics ${ }^{\circledR}$, following the guidelines laid previously ${ }^{21,22}$. The electromagnetic field resonantly coupled to the microsphere (for $d=0$ ) is confined in a few micrometers below the surface. We show in Fig. 3 the calculated spectra for two close values of the applied voltage (V), ideally corresponding to $n=n_{T E}$ (twist) equal to 1.5 and 1.52 . The dips in the transmitted spectra are at less than $-48 \mathrm{~dB}$, with $3 \mathrm{~dB}$ line-widths of only $6 \mathrm{pm}(750 \mathrm{MHz})$, when calculated with wavelength step as narrow as $1 \mathrm{pm}$. Hence, the loaded quality factor Q of the filter is approximately $2.6 \cdot 10^{4}$ and remains virtually unchanged when the filter is electrically tuned.

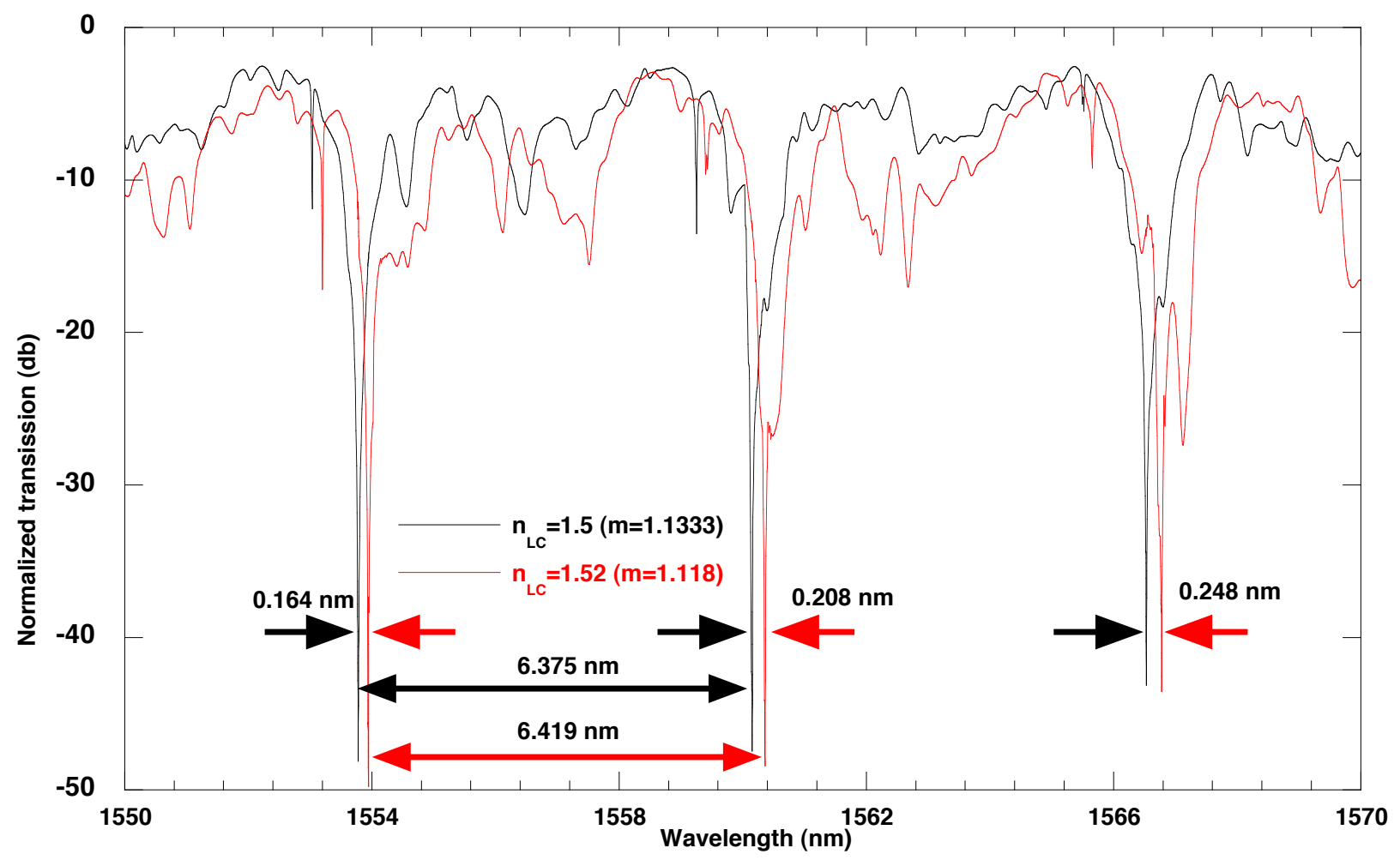

Figure 3. Computed normalized transmission as a function of the applied voltage. Thin black line at ground state where $n=n_{T E}($ twist $)=1.5$, red line for $n=n_{T E}($ twist $)=1.52$. Arrows show spectral shift for increasing $n=n_{T E}($ twist $)$.

\subsection{Analytical model}

Elastic scattering intensities from a sapphire microsphere in a LC excited by a glass optical waveguide is calculated by using GLMT. The method requires to approximate the actual waveguide index profile as a cylindrical waveguide in which a Gaussian beam propagates ${ }^{23}$. It also requires that the LC is not confined only in the cavity shown in Figure 2 (b) but everywhere around the sphere. Figure 4 shows the schematic, for which the GLMT elastic scattering calculations are performed. 


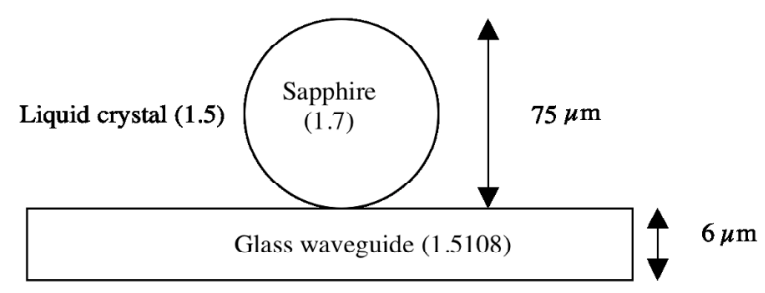

Figure 4. The schematic used for the GLMT calculations.

Figure 5 shows the calculated elastic scattering spectra at $0^{\circ}$ (transmission) for transverse electric (TE) polarizations. Three sets of modes are visible with a mode spacing of $\Delta \lambda=6.18 \mathrm{~nm}$, which agrees well with the value obtained from the following:

$$
\Delta \lambda=\lambda_{0} \lambda_{\text {glass }} \frac{\operatorname{tg}^{-1}\left(\left(m^{2}-1\right)^{\frac{1}{2}}\right)}{\left(2 \pi s\left(m^{2}-1\right)^{\frac{1}{2}}\right)}
$$

where $\lambda_{0}$ is the wavelength in vacuum, $\lambda_{\text {glass }}$ is the wavelength in glass, $s$ is the radius of the microsphere, and $m$ is the normalized refractive index of the microsphere with respect to the liquid crystal surroundings. The refractive index of the liquid crystal is taken to be 1.5 and 1.5005 , corresponding to the normalized refractive indices $m$ of 1.1333 and 1.1330 , respectively. For the calculations, this $0.03 \%$ increase of the refractive index of the liquid crystal, results in a proportional spectral shift of $0.49 \mathrm{~nm}$ as indicated in Figure 5.

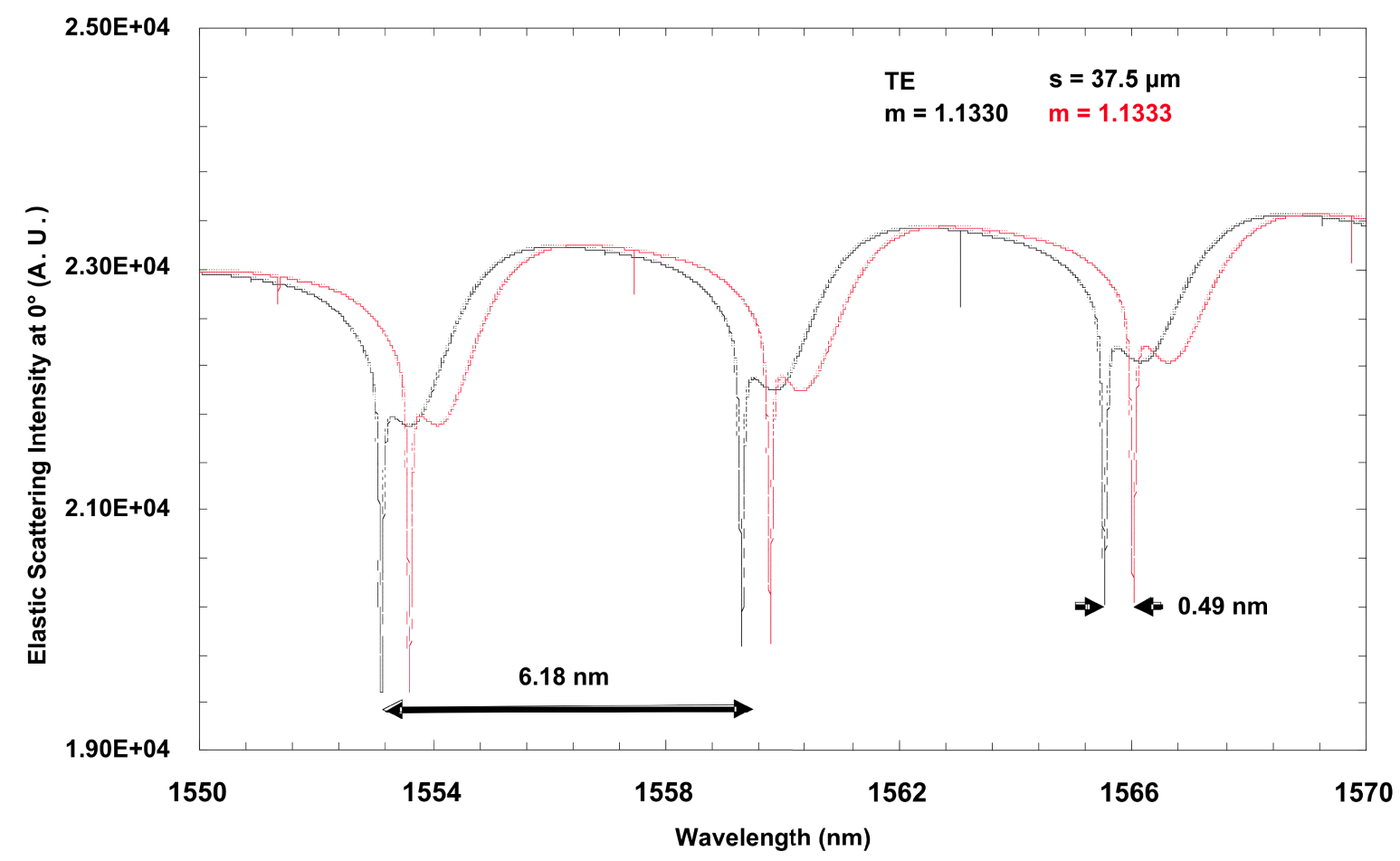

Figure 5. Elastic scattering spectra at $0^{\circ}$ (transmission) for the TE polarization. 


\section{DISCUSSION AND CONCLUSIONS}

The comparison of the analytical solutions found by Generalized Lorenz-Mie Theory (GLMT) and numerically by a Finite Element Method (FEM) are in excellent agreement in term of spectra shapes, absolute wavelength of dips and the mode spacing as one can easily see by comparing figures 3 and 5. However, the amount of spectral shift for increasing refractive index of the isotropic refractive index differs significantly. The main reason is that the FEM analysis considers the actual geometry, where the LC is only in recessed reservoir and is in contact with the sphere by $\approx 10 \%$ on both to the overall equatorial circumference and total surface. Conversely, the GLMT considers a sphere fully submerged in the LC crystal, thus overestimating its tuning effect. With an applied control voltage, in the FEM model, the WGM's can shift to red (effective radius increase) or blue (effective refractive index decrease), whereas in the GLMT model the WGM's only shift to blue (effective refractive index decrease).

\section{REFERENCES}

[1] Lord Rayleigh, "The problem of the whispering gallery", Scientific Papers (Cambridge University), 5, 617-620 (1912).

[2] Serpengüzel, A., Arnold, S., and Griffel, G., "Excitation of Resonances of Microspheres on an Optical Fiber," Opt. Lett. 20(7), 654-656 (1995).

[3] Serpengüzel, A., Arnold, S. Griffel, G. and Lock, J. A., "Enhanced Coupling to Microsphere Resonances with Optical Fibers," J. Opt. Soc. Am. B 14(4), 790-795 (1997).

[4] Griffel, G., Arnold S., Taskent D., Serpengüzel A., Connolly, J., and Morris N., "Morphology Dependent Resonances of a Microsphere/Optical Fiber System," Opt. Lett. 21(10), 695-697 (1996).

[5] Cai, M., Hunziker, G. and Vahala, K., "Fibre optic add-drop device based on a silica microsphere-whispering gallery mode system," IEEE Photon. Technol. Lett. 11(6), 686-687 (1999).

[6] Panitchob, Y., Senthil Murugan, G., Zervas, M. N., Horak, P., Berneschi, S. Pelli, S., Nunzi Conti, G. and Wilkinson, J. S. "Whispering gallery mode spectra of channel waveguide coupled microspheres," Opt. Express 16(15), 11066 (2008).

[7] Cai, M., Painter, O. and Vahala K.J., "Fiber-coupled microsphere laser,” Opt. Lett. 25(19), 1430-1432 (2000).

[8] Bilici, T., Isci, S., Kurt, A., and Serpengüzel, A., "Microsphere Based Channel Dropping Filter with Integrated Photodetector," IEEE Photon. Technol. Lett. 16(2), 476-478 (2004).

[9] Yilmaz, Y.O., Demir A., Kurt A., and Serpengüzel, A., "Optical Channel Dropping with a Silicon Microsphere," IEEE Photon. Technol. Lett. 17(8), 1662-1664 (2005).

[10] Tapalian, H.C., Laine, J.P., Lane P.A., “Thermooptical switches using coated microsphere resonators”, IEEE Photon. Technol. Lett. 14(8), 1118-1120 (2002).

[11] Chang, R.K. and Campillo, A.J. (Eds.) "Optical processes in microcavities," World Scientific, Singapore, (1996).

[12] Spillane, S.M., Kippenberg, J.T. and Vahala, K.J., "Ultralow threshold Raman laser using a spherical dielectric microcavity," Nature 415(6872), 621-623 (2002).

[13] Yüce E., Gürlü O., and Serpengüzel A., "Optical Modulation with Silicon Microspheres," IEEE Photon. Technol. Lett. 21(20), 1481-1483 (2009).

[14] d'Alessandro, A., Donisi, D., Asquini, R., Beccherelli, R., De Sio, L., and Umeton C., "Tunable integrated optical filter made of a glass ion-exchanged waveguide and an electro-optic composite holographic grating," Opt. Express 16(13), 9254-9260 (2008).

[15] Zografopoulos, D. C., Kriezis, E. E., Bellini, B., and Beccherelli, R. "Tunable one-dimensional photonic crystal slabs based on preferential etching of silicon-on-insulator," Opt. Express 15(4), 1832-1844 (2007).

[16] Tasolamprou, A. C., Bellini, B., Zografopoulos, D. C., Kriezis, E. E., and Beccherelli, R., "Tunable optical properties of silicon-on-insulator photonic crystal slab structures," Journal of the European Optical Society - Rapid Publications 4, 09017 (2009).

[17] Gilardi, G., Donisi D., Serpengüzel A., and Beccherelli R., "Liquid crystal tunable filter based on sapphire microspheres," Opt. Lett. 34(21), 3253-3255 (2009).

[18] Piegdon, K.A., Offer, M., Lorke, A., Urbanski, M., Hoischen, A., Kitzerow, H.-S., Declair, S., Förstner J., Meier, C. "Self-assembled quantum dots in a liquid-crystal-tunable microdisk resonator," Physica E (2010) doi:10.1016/j.physe.2009.12.051 
[19] Lock, J. A. “An Improved Gaussian Beam Scattering Algorithm,” Appl. Opt. 34(3), 559-570 (1995).

[20] Bellini, B., d'Alessandro, A., and Beccherelli R., "A method for butt-coupling optical fibres to liquid crystal planar waveguides," Optical Materials 29, 1019-1022 (2007).

[21] Bellini, B., Beccherelli, R., " Modelling, design and analysis of liquid crystal waveguides in preferentially etched silicon grooves," Journal of Physics D: Applied Physics 42(4), 045111 (2009).

[22] Donisi, D., Bellini, B., Beccherelli, R., Asquini, R., Gilardi, G., Trotta, M., and d'Alessandro, A.," A Switchable Liquid-Crystal Optical Channel Waveguide on Silicon," IEEE Journal of Quantum Electronics 46(5), 762-768 (2010).

[23] Gouesbet, G. Lock, J. A., "Rigorous justification of the localized approximation to the beam-shape coefficients in generalized Lorenz-Mie theory II. Off-axis beams,” J. Opt. Soc Am. A. 11(9), 2516-2525 (1994). 